\title{
Anesthetic Management of a Patient With Multiple Sclerosis
}

\author{
Naohiro Ohshita, DDS, PhD,* Shoko Gamoh, DDS, PhD, $\dagger$ Masahiko Kanazumi, DDS, \\ PhD, $*$ Masahiro Nakajima, DDS, PhD, $\neq$ Yoshihiro Momota, DDS, PhD, $*$ and Yasuo M. \\ Tsutsumi, MD, PhDS \\ *Department of Anesthesiology, †Department of Oral Radiology, and łSecond Department of Oral and Maxillofacial Surgery, Osaka Dental \\ University, Osaka, Japan, and §Department of Anesthesiology, Tokushima University, Tokushima, Japan
}

A 54-year-old woman diagnosed with multiple sclerosis (MS) at the age of 19 years was scheduled to undergo temporomandibular joint mobilization. She was currently in a remission phase from her MS but with persistent sequelae, including impaired eyesight and muscle weakness of the limbs. In addition, the blood vessels in her upper limbs were compromised by the formation of internal shunts secondary to vascular prosthesis replacements for plasma exchange therapy in MS. After a previous joint mobilization surgery, her temporomandibular joint developed adhesions with resultant trismus. One of the adverse effects of general anesthesia can be exacerbations of MS symptoms. Minimizing mental and physical stress caused by surgical and anesthetic procedures and maintenance of stable body temperature are important considerations. Awake intubation was performed under sedation with midazolam and fentanyl. After intubation, anesthesia was induced with propofol, remifentanil, and rocuronium. Maintenance of anesthesia was achieved with oxygen- $\mathrm{N}_{2} \mathrm{O}$-sevoflurane, remifentanil, fentanyl, and rocuronium. In this case, no adverse events occurred intraoperatively. However, the patient experienced lingering weakness of the limbs in the postoperative period, and activities of daily living of the patient were affected.

Key Words: Multiple sclerosis; Demyelinating encephalomyelitis; Trismus.

$\mathbf{M}$ ultiple sclerosis (MS) is a demyelinating disease of the central nervous system characterized by varying initial symptoms such as visual impairment, bowel/bladder dysfunction, trigeminal neuralgia, cognitive problems, gait disorder, and motor paralysis. We report a case of a patient with MS who underwent temporomandibular joint (TMJ) mobilization under general anesthesia.

\section{CASE REPORT}

A 54-year-old woman (height, $155 \mathrm{~cm}$; weight, $45 \mathrm{~kg}$ ) with MS was scheduled for TMJ mobilization under general anesthesia. The patient was diagnosed with MS at the age of 19 years, and reportedly was in remission since the age

Received August 11, 2016; accepted for publication December 27, 2016.

Address correspondence to Naohiro Ohshita, Department of Anesthesiology, Osaka Dental University, 1-5-17, Ohtemae, Chuo, Osaka, 540-0008, Japan; oshita@cc.osaka-dent.ac.jp.

Anesth Prog 64:97-101 2017 | DOI 10.2344/anpr-64-02-10

(C) 2017 by the American Dental Society of Anesthesiology of 35 years, after multiple episodes of remissions and exacerbations. The MS was treated mainly with steroidpulse, immunoglobulin, and plasma exchange therapy. Although the MS was in a remission phase, she had persisting after-effects, including impaired eyesight due to steroid-induced glaucoma and cataract, and muscle weakness of limbs. She used to walk with the help of a cane, but her activities of daily living had since decreased. In addition, the blood vessels of both upper extremities and the left lower extremity were compromised as a result of formation of internal shunts secondary to vascular prosthesis replacement for plasma exchange therapy and steroid pulse therapy. Her arms and legs appeared edematous. Other medical history included gastroesophageal reflux and history of hypotension. The gastroesophageal reflux was treated with sulpiride (a dopamine antagonist antipsychotic), lansoprazole (a proton pump inhibitor), mosapride citrate (a 5- $\mathrm{HT}_{4}$ gastroprokinetic), and polaprezinc (a mucosal protective agent), and hypotension was treated with amezinium metilsulfate (a sympathomimetic drug with $\alpha$ and $\beta$ effects). 


\begin{tabular}{|c|c|c|c|c|c|c|c|c|c|}
\hline & $\begin{array}{c}\text { Affer entering } \\
\text { Operation room }\end{array}$ & $\begin{array}{c}\text { After } \\
\text { induction }\end{array}$ & $\begin{array}{c}\text { During } \\
\text { intubation }\end{array}$ & $\begin{array}{c}\text { After } \\
\text { intubation }\end{array}$ & $\begin{array}{c}\text { During } \\
\text { Operation (1) }\end{array}$ & $\begin{array}{c}\text { During } \\
\text { Operation (2) }\end{array}$ & $\begin{array}{c}\text { During } \\
\text { Operation (3) }\end{array}$ & $\begin{array}{c}\text { During } \\
\text { Operation (4) }\end{array}$ & $\begin{array}{c}\text { After } \\
\text { Extubation }\end{array}$ \\
\hline $\begin{array}{c}\text { Blood pressure } \\
\text { (mmHg) }\end{array}$ & $130 / 70$ & $98 / 52$ & $148 / 72$ & $102 / 58$ & $80 / 50$ & $92 / 55$ & $118 / 55$ & $108 / 55$ & $130 / 70$ \\
\hline $\begin{array}{c}\text { Mean blood } \\
\text { pressure } \\
\text { (mmHg) }\end{array}$ & 90 & 67 & 97 & 73 & 60 & 67 & 76 & 73 & 90 \\
\hline $\begin{array}{c}\text { Medication } \\
\text { by } \\
\text { vasopressors }\end{array}$ & \multicolumn{7}{|c|}{ Ephedrine $\uparrow$} \\
\hline
\end{tabular}

Arrows indicate the timing each medication was administered. Ephedrine was given at $8 \mathrm{mg} / \mathrm{bolus}$, phenylephrine at $0.05 \mathrm{mg} / \mathrm{bolus}$, and dopamine at $2 \mu \mathrm{g} / \mathrm{kg} / \mathrm{min}$.

The treatment for MS was symptomatic and comprised azathioprine, ethyl icosapentate (an omerga-3 fatty acid derivative), limaprost alfadex (a prostaglandin E1 derivative, which increases nerve root blood flow), xalatan eye lotion, vitamins, and an ATP preparation. Analgesic drugs included various medications, such as celecoxib, acetaminophen, and pregabalin. She had a history of allergy to loxoprofen sodium (a propionic acid derivative NSAID), rebamipide (a quinolinone for mucosal protection), cefcapene pivoxil $\mathrm{HCl}$ (a thirdgeneration cephalosporin), and crustaceans. Due to these multiple allergies, she typically carried an epinephrine auto-injector as an emergency drug.

Her past surgical history included an insertion of intraocular lens for cataract in her 20s, an open reduction of a femoral fracture under spinal anesthesia at the age of 47 years, and TMJ mobilization under general anesthesia at the age of 52 years. The patient lived in another prefecture and underwent a previous TMJ mobilization operation in another hospital. However, her TMJ adhered to the surrounding tissue immediately after the first TMJ mobilization surgery, and her trismus never improved. After the first TMJ mobilization, the patient had sustained damage to the inner ear due to surgical error, leading to right-sided deafness.

The findings of the preoperative examinations were mostly normal with a few exceptions. Laboratory abnormalities included a hemoglobin value of $10.5 \mathrm{~g} /$ $\mathrm{dL}$ (normal 11.3-15.2 $\mathrm{g} / \mathrm{dL}$ ) and a total bilirubin value of $1.7 \mathrm{mg} / \mathrm{dL}$ (normal $0.2-1.2 \mathrm{~g} / \mathrm{dL}$ ). The electrocardiogram indicated a left axis deviation and an inverted $\mathrm{T}$ in leads III and V1. The chest radiograph, however, revealed a normal cardiothoracic ratio of $43.2 \%$. Due to poor expiration, the patient could not undergo pulmonary function test; however, evaluation of room air arterial blood gas was essentially normal. Axillary temperature preoperatively ranged from 35.0 to $36.2^{\circ} \mathrm{C}$. After admission a day before surgery, a catheter was inserted into the right internal jugular vein of the patient by the division of neurosurgery at an affiliated hospital.

On the day of the operation, we continued only amezinium metilsulfate and the patient's vital signs were as follows: blood pressure (mean), 130/70 (90) $\mathrm{mmHg}$; heart rate, 58 beats per minute with normal sinus rhythm; body temperature, $36.4^{\circ} \mathrm{C} ; \mathrm{SpO}_{2}, 100 \%$ on room air. Due to the limited mouth opening, we opted for awake intubation with sedation. Moderate sedation was obtained with midazolam $(1.5 \mathrm{mg})$, fentanyl $(100$ $\mu \mathrm{g})$ via the internal jugular catheter. Lidocaine $1 \%$ including 1:100,000 epinephrine was applied for topical anesthesia of nasal mucosa and $4 \%$ lidocaine was sprayed for vocal cord anesthesia. Intubation was successfully carried out with bronchofiberscope. After intubation, $50 \mathrm{mg}$ of propofol and $25 \mathrm{mg}$ of rocuronium were given for induction of anesthesia. Subsequently, anesthesia was maintained with $1.5 \%$ sevoflurane in air and oxygen and $0.1 \mu \mathrm{g} / \mathrm{kg} / \mathrm{min}$ remifentanil. Nitrous oxide $\left(\mathrm{N}_{2} \mathrm{O}\right) 50 \%$ in oxygen was added for the final hour of the operation with reduction in sevoflurane dosage. Additional intraoperative medications included $20 \mathrm{mg}$ rocuronium at the oral surgeon's request to facilitate an unimpeded TMJ surgery and $3.3 \mathrm{mg}$ dexamethasone. Fentanyl $25 \mu \mathrm{g}$ and acetaminophen $15 \mathrm{mg} / \mathrm{kg}$ were administered by intravenous injection for postoperative analgesia. Metoclopramide $10 \mathrm{mg}$ was also administered to prevent postoperative vomiting and nausea. The effect of rocuronium was reversed by atropine $(1 \mathrm{mg})$ and neostigmine $(2 \mathrm{mg})$ at the end of the operation. We assessed neuromuscular function by monitoring TOF prior to reversal and extubated after confirming proper recovery from muscle relaxation.

During the operation, vital signs were stable with a heart rate ranging between 50 and 70 beats per minute without arrhythmia, rectal temperature ranging from 36.4 to $37.0^{\circ} \mathrm{C}$, and $\mathrm{SpO}_{2}$ of $100 \%\left(\mathrm{FiO}_{2}=0.3\right)$. An exception was blood pressure, which ranged from $80 / 50$ (60) to $148 / 72$ (97) $\mathrm{mmHg}$ (see the Table). Blood 
pressure was stabilized with a total of $24 \mathrm{mg}$ ephedrine, $0.2 \mathrm{mg}$ phenylephrine, and continuous administration of dopamine at $2 \mu \mathrm{g} / \mathrm{kg} / \mathrm{min}$. The anesthesia procedure was completed in 3 hours and 45 minutes (operation time 2 hours and 10 minutes) with a total fluid infusion volume of $1500 \mathrm{~mL}$ and a total urine volume of $580 \mathrm{~mL}$ with minimal bleeding. Postoperatively, she had no alteration in respiratory function but loss of strength of the legs for 1 week. She could walk with assistance, but needed time to stand up by herself. Her axillary temperature ranged between 36.3 and $37.2^{\circ} \mathrm{C}$ during the 7 days after operation. Temperature rose to $37.4^{\circ} \mathrm{C}$ during days 10 and 11 after the operation. Various laboratory tests did not differ from baseline including white blood cell count and leukocyte fraction except for the maximum value of C-reactive protein $0.37 \mathrm{mg} / \mathrm{dL}$ (normal $0-0.03 \mathrm{mg} / \mathrm{dL}$ ) during the perioperative period. The patient was discharged 1 month after the operation.

\section{DISCUSSION}

MS is a type of demyelinating encephalomyelitis of the central nervous system with symptoms affecting sensory and/or motor nerves that typically undergo a relapsing/ remitting course. Neurological dysfunction characterizes the common symptoms of MS, which include paralysis, sensory disturbances, autonomic disturbances, lack of coordination, and visual impairment. ${ }^{1}$ This patient's presenting symptoms were low vision from optic neuritis followed by left hemiparesis. The cause of MS is not clear, but it is considered an autoimmune disease resulting in myelin destruction. Exacerbating factors include environmental factors, including viral infections, smoking, decreased sun exposure, and vitamin D deficiency. ${ }^{6}$ While MS is not hereditary, having a parent or sibling with MS increases an individual's risk. ${ }^{6}$ Triggers of MS include stressful situations, such as infection, inflammation, and fever. ${ }^{6}$ The disease mainly affects women between 10 and 60 years of age, with the greatest incidence of patients between 20 and 40 years of age. ${ }^{1}$ Our patient developed MS at the age of 19 years, and the disease apparently showed remission since the age of 35 years. However, for the first 15 years since diagnosis, she had frequent relapses. Her activities of daily living had gradually worsened, and the current after-effects of MS included muscle weakness, chronic fatigue, autonomic disturbances, and visual impairment. Literature on the most appropriate method of general anesthesia in MS is generally limited to case reports and describe the role of stress and anesthesia in inducing exacerbations of central nervous system symptoms. ${ }^{2-6}$ However, many clinicians believe that general anesthesia causes fewer exacerbations and is superior to neuraxial techniques. ${ }^{1}$ Surgical procedures can affect physical condition when the state of MS is unstable. In this case, the patient experienced an MS relapse after undergoing an operation inserting an intraocular lens.

For administering anesthetic drugs in patients with MS, caution is needed when using neuromuscular blocking agents. ${ }^{1-6}$ In these patients, resistance to nondepolarizing neuromuscular blocking agents may develop due to increased number of acetylcholine receptors produced by denervation. ${ }^{5}$ In addition, muscle weakness and decreased muscle mass are associated with increased sensitivity to neuromuscular blocking agents. ${ }^{1,5,6}$ Since this protocol required use of the lowest anesthetic dose and was intended to maintain anesthesia without additional administration of muscle relaxant (rocuronium), sevoflurane was chosen as the most appropriate agent. However, we administered additional rocuronium $(20 \mathrm{mg})$ according to the oral surgeon's request to facilitate an unimpeded TMJ surgery. Muscle relaxant with a relatively short half-life would be safer to use minimizing the complication of respiratory dysfunction. The patient recounted that she could tell what surgeons said in the last hour during surgery of the first TMJ mobilization. The anesthetics may have been insufficient at this surgery due to efforts to limit anesthetic drug exposure. Therefore, we used both $50 \% \mathrm{~N}_{2} \mathrm{O}$ and $0.7 \%$ sevoflurane for the purpose of reducing the latter dosage while preserving the amnestic effect during the final hour of the operation. $\mathrm{N}_{2} \mathrm{O}$ administration to prevent anesthetic awareness is controversial ${ }^{7}$; however, $\mathrm{N}_{2} \mathrm{O}$ would be effective for maintenance of anesthesia during minimally invasive operations and is not associated with the risk of developing $\mathrm{MS}^{8}{ }^{8}$ Concerning body temperature, the possibility of development of hyperthermia should be considered. ${ }^{1,4-6}$ Heat exposure blocks conduction in demyelinated axons and can trigger recurrence of MS, and body temperature should be closely monitored and controlled during the perioperative period. ${ }^{1,6}$ The patient had been avoiding any conditions that may lead to hyperthermia. To ensure a stable body temperature, she was careful not to go into hot springs, for instance. As hypothermia also induces shivering, we maintained her body temperature at approximately $37^{\circ} \mathrm{C}$ by using techniques for cooling and warming the body during the operation. We administered midazolam (approximately $0.03 \mathrm{mg} / \mathrm{kg}$ ) for awake intubation in this present case. Additionally, we were concerned that an increase in the quantity of midazolam might worsen potential perioperative adverse events in respiratory function and delayed emergency from anesthesia. However, benzodiazepines, including midazolam, have a great advantage in MS patients with their minimal effect on thermoregulatory control ${ }^{4}$ and the prevention of postoperative 
shivering, ${ }^{10}$ so that a low dose might be considered to be given closer to the end of anesthesia. ${ }^{9}$ The patient's axillary temperature ranged between 36.3 and $37.2^{\circ} \mathrm{C}$ during the 7 days after operation, which could be attributed to inflammation (but not evident infection). This would be within the range of a slight fever that may be expected in the postoperative period.

There was no alteration in respiratory function after general anesthesia, but patients with MS tend to show loss of strength, sometimes very pronounced, of the lower extremities after general anesthesia. This patient showed a $20 \%$ recovery of the normal muscular strength after 1 day and $70 \%$ recovery after 5 days. The experienced loss of strength in the legs was probably related to symptoms of MS despite special attention to use of anesthetic agents. Considering the etiology of the case, we considered referring the patient to an affiliated medical hospital rather than our dental hospital for postoperative care, but she always experienced loss of muscular strength after operations and was accustomed to this weakness after general anesthesia. Previous reports indicate that small elevations of temperature can block conduction in marginally functioning demyelinated axons, ${ }^{10}$ and alterations as small as $1{ }^{\circ} \mathrm{C}$ may trigger exacerbations in MS. The muscle strength could be sensitive to a sharp "change" in body temperature, irrespective of the absolute body temperature. She was treated with only azathioprine for MS, and we administered minimal doses of dexamethasone during the operation and on postoperation day 1 . It is not clear if the corticosteroid administration had a positive effect on recovery of muscular strength in the legs.

In the present case, a central line was placed in the right internal jugular vein before the operation because the patient's blood vessels in the bilateral upper limbs and the left lower limb were compromised as a complication of the therapeutic procedures for MS. In the early treatment of her MS, she initially had a cannula inserted into the left femoral vein by venous cut-down procedure for plasma exchange therapy. However, that vein became unusable, and development of vascular shunts and subsequent vascular prosthesis replacements were made on the left forearm in turn. In addition, she had been treated by ordinary drip infusion of steroids so many times that intravenous access of the right upper limb was also so severely compromised that a venous cut-down procedure was performed as a last attempt in this arm. In the first operation TMJ mobilization operation, the patient underwent a peripheral venous cut-down procedure since no one was able to insert a standard peripheral intravenous catheter. She had been following up by the division of nephrology for extremity edema, which was not associated with organic disease of the kidney or heart.
In addition, the patient suffered from trismus, which necessitated nasal intubation using bronchofiberscope under conscious sedation. In this case, the cause of trismus would be due to adhesion of articular disc, which was returned easily to the presurgical state after the initial surgery. This severe condition was not specific to MS, but among patients presenting TMD symptoms, the prevalence of the patients with MS was reported to be significantly higher than the control group, irrespective to the severity of MS. ${ }^{11}$ We referred to the difficult airway management algorithm, and planned the intubation accordingly.

Another important aspect for the anesthesiologist is the variable degree of hemodynamic instability. Hypotension that occurs especially under regional anesthesia is frequently resistant to hydration and vasopressors. ${ }^{6}$ In addition, patients with autonomic dysfunction can demonstrate an exaggerated response to vasodilators and $\alpha$-sympathomimetics. ${ }^{1}$ In the present case, the patient was treated for intraoperative hypotension during the surgical procedure. We had to balance the amount of intravenous fluid administration to ensure adequate renal perfusion and urine output while at the same time maintaining adequate blood pressure and limiting exacerbation of the extremity edema. We eventually chose a dopamine infusion to maintain hemodynamic stability, and we did not experience exaggerated response to $\alpha$-sympathomimetics.

In conclusion, we present a case of general anesthesia for TMJ mobilization surgery in a patient with MS. This patient with MS was safely managed with special attention given to perioperative stress, hyperthermia, and anesthetic agents selected. Despite these measures, loss of strength in the lower limbs occurred for 1 week following surgery and anesthesia.

\section{REFERENCES}

1. Nozari A, Bagchi A, Saxena R, Bateman BT. Neuromuscular disorders and other genetic disorder. In: Miller RD, ed. Miller's Anesthesia. Vol. 1, 8th ed. New York, NY: Churchill Livingstone; 2015:1266-1286.

2. Casati V, Tesler UF, Novelli E, et al. Off-pump coronary surgery in a patient with multiple sclerosis. Heart Lung Vessel. 2013;5:179-182.

3. Sahin L, Korkmaz HF, Sahin M, Aydin T, Toker S, Gulcan E. Desflurane anaesthesia in a patient with multiple sclerosis in total hip replacement. Arch Med Sci. 2010;6:984986.

4. Ceyhan A, Uyar ET, Gencay IY, Gunal SE. Anesthesia in multiple sclerosis and obstructive sleep apnea: case report and literature review. J Res Med Sci. 2011;16:828-835.

5. Acar A, Nuri Deniz M, Erhan E, Ugur G. Anesthetic technique in a patient with multiple sclerosis scheduled for 
laparoscopic nephrectomy for a renal tumor: a case report. Anesth Pain Med. 2013;2:138-140.

6. Makris A, Piperopoulos A, Karmaniolou I. Multiple sclerosis: basic knowledge and new insights in perioperative management. J Anesth. 2014;28:267-278.

7. de Vasconcellos K, Sneyd JR. Nitrous oxide: are we still in equipoise? A qualitative review of current controversies. $\mathrm{Br}$ J Anaesth. 2013;111:877-885.

8. Hedström AK, Hillert J, Olsson T, Alfredsson L. Exposure to anesthetic agents does not affect multiple sclerosis risk. Eur J Neurol. 2013;20:735-739.
9. Grover VK, Mahajan R, Yaddanapudi LN, Sudarshana $\mathrm{HG}$, Gill KD. Efficacy of midazolam in preventing postoperative shivering. Int J Clin Pharmacol Ther. 2002;40:534-536.

10. Siemkowicz E. Multiple sclerosis and surgery. Anaesthesia. 1976;31:1211-1216.

11. Carvalho LS, Matta AP, Nascimento OJ, Guimarães AS, Rodrigues LR. Prevalence of temporomandibular disorders symptoms in patients with multiple sclerosis. Arq Neuropsiquiatr. 2014;72:422-425. 